\title{
Nonnormality and the localized control of extended systems
}

\author{
Roman O. Grigoriev, Andreas Handel \\ School of Physics, Georgia Institute of Technology, Atlanta, GA 30332-0430
}

(November 15, 2018)

\begin{abstract}
The idea of controlling the dynamics of spatially extended systems using a small number of localized perturbations is very appealing - such a setup is easy to implement in practice. However, when the distance between controllers generating the perturbations becomes large, control fails due to increasing sensitivity of the system to noise and nonlinearities. We show that this failure is due to the fact that the evolution operator for the controlled system becomes increasingly nonnormal as the distance between controllers grows. This nonnormality is the result of control and can arise even for systems whose evolution operator is normal in the absence of control.
\end{abstract}

PACS numbers: 02.30.Yy, 05.45.Gg

Control of spatially extended systems has recently emerged as a problem of fundamental interest as well as significant technological importance. Numerous investigations have shown the possibility of controlling a system by applying feedback at every point in space. However, the practical implementation of such control algorithms is often prohibitively expensive and sometimes impossible. As a result, increasing attention is being devoted to localized control, where feedback is applied only at a few spatial locations. Previous studies have shown that the minimal number of such control locations depends both on the symmetry of the system [1] and the strength of noise [2,3].

The failure of localized control for large separation between controllers was attributed to the phenomenon of nonnormality in the evolution operator 3. For nonnormal systems, stability becomes a poor predictor of short term dynamics [4]. Strong nonnormality, which makes the system extremely sensitive to noise, was previously identified as the mechanism that provokes the transition to turbulence in uncontrolled systems, such as pipe or channel flows [5] [6]. The latter systems are nonnormal due to a large mean flow, whereas generic extended systems have no mean flow and are normal for typical boundary conditions. In these normal systems, nonnormality arises as a result of control. Studies of nonnormality caused by localized control [3] have so far been limited to the interplay between nonlinearity and the noise amplification due to nonnormality, rather than the emergence of nonnormality itself. Our goals here are to investigate how nonnormality arises in an originally normal system and study how nonnormality leads to noise amplification, which determines the limits of localized control.

Let us consider the Ginzburg-Landau equation (GLE)

$$
\dot{\phi}(x, t)=\phi(x, t)+\phi^{\prime \prime}(x, t)-\phi^{3}(x, t)
$$

as a prototypical system. Although simple enough to allow analytical treatment, the GLE describes a very generic reaction-diffusion system. We therefore expect the main results of the following analysis to apply to most extended dynamical systems of this type.

The unbounded system (11) possesses an unstable uniform steady state, $\phi=0$. Our control objective is to make this state stable. The symmetry of an unbounded system requires at least two independent controllers in order to control a uniform target state [1], so we will break the symmetry by imposing certain boundary conditions. Specifically, we will require that $\phi$ vanishes on one of the boundaries, e.g., $\phi(0, t)=0$, reducing the minimal number of controllers to one. The only controller will be placed at the opposite boundary, $x=l$. With this arrangement the length $l$ of the system plays the role of the distance between multiple controllers in a system of larger size. We choose the feedback law to be of the form

$$
\phi^{\prime}(l, t)=\int_{0}^{l} K(y) \phi(y, t) d y,
$$

such that the control signal depends on the state $\phi$ of the system on the whole domain (this condition can be easily relaxed). The feedback gain $K(y)$ describes how each point inside the domain contributes to the feedback.

The spectrum of the unperturbed linearized system is discrete with eigenvalues and eigenfunctions given by

$$
\lambda_{n}=1-q_{n}^{2}, \quad f_{n}(x)=\sin \left(q_{n} x\right),
$$

where $q_{n}=(n-1 / 2) \pi / l$ and $n=1,2, \cdots$. These eigenfunctions are normal, as expected, and form a convenient basis for the stability analysis of the perturbed system. Dropping the cubic term in (11) and projecting the remainder onto the basis $\left\{f_{n}\right\}$ we obtain

$$
\dot{\Phi}_{n}=\lambda_{n} \Phi_{n}-(-1)^{n} \sum_{m=1}^{\infty} K_{m} \Phi_{m} \equiv(M \Phi)_{n},
$$

where $\Phi_{n}$ and $K_{n}$ are the Fourier coefficients of $\phi$ and $K$

$$
\begin{aligned}
\Phi_{n} & =\frac{2}{l} \int_{0}^{l} \phi(x) \sin \left(q_{n} x\right) d x, \\
K_{n} & =\frac{2}{l} \int_{0}^{l} K(x) \sin \left(q_{n} x\right) d x,
\end{aligned}
$$




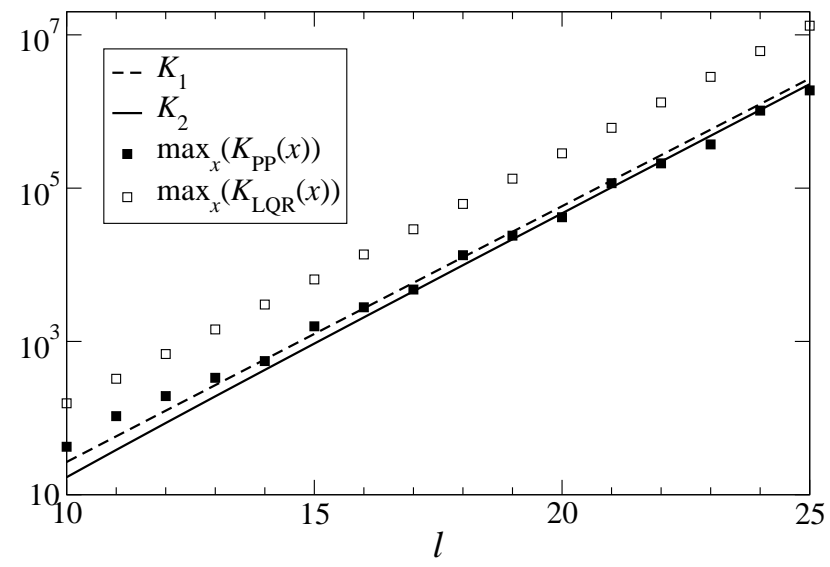

FIG. 1. Strength of feedback as a function of the size $l$ of the system. The maximum of $K(x)$ in real space is compared with the Fourier coefficients $K_{1}$ and $K_{2}$ calculated using (9).

and

$$
M=\left(\begin{array}{cccc}
\lambda_{1}+K_{1} & K_{2} & K_{3} & \cdots \\
-K_{1} & \lambda_{2}-K_{2} & -K_{3} & \cdots \\
K_{1} & K_{2} & \lambda_{3}+K_{3} & \cdots \\
\vdots & \vdots & \vdots & \ddots
\end{array}\right)
$$

The control problem for the PDE (1) is thus reduced to finding an infinite set of coefficients $K_{m}$, which will make the matrix $M$ stable.

It turns out that the structure of $M$ simplifies the problem remarkably. Suppose we take an $n \times n$ truncation of $M$ by discarding all rows and columns except the first $n$. Setting the eigenvalues of the truncated matrix, $M_{n}$, to a sequence $\lambda_{1}^{\prime}, \lambda_{2}^{\prime}, \cdots, \lambda_{n}^{\prime}$ is then equivalent to solving a system of $n$ equations linear in $K_{m}$ 's. In particular, one can change the first $m=1, \cdots, s$ eigenvalues from $\lambda_{m}$ to $\lambda_{m}^{\prime}$ and leave the rest unchanged by setting

$$
K_{m}^{(n)}=-\frac{\prod_{p=1}^{s}\left(\lambda_{m}-\lambda_{p}^{\prime}\right)}{\prod_{p=1}^{m-1}\left(\lambda_{p}-\lambda_{m}\right) \prod_{p=m+1}^{s}\left(\lambda_{m}-\lambda_{p}\right)},
$$

for $m \leq s$ and zero otherwise. Since the right-handside does not depend on the size of the truncated matrix $M_{n}$, the result also holds for the full matrix $M$, so we can drop the index $n$. This is a very important result, because it allows us to calculate Fourier coefficients of any stabilizing feedback gain. It also allows us to determine how these coefficients scale with the size of the system $l$. Substituting (3) into (7) and after some algebra we obtain

$$
K_{m}=-\left(\frac{l}{\pi}\right)^{2(s-1)} \frac{(2 m-1) \prod_{p=1}^{s}\left(\lambda_{m}-\lambda_{p}^{\prime}\right)}{(s+m-1) !(s-m) !} .
$$

To make the matrix $M$ stable we only need to change the first $s$ positive eigenvalues, where $s$ is equal to the integer part of $l / \pi+1 / 2$. In particular, in the limit $\lambda_{1}^{\prime}=$

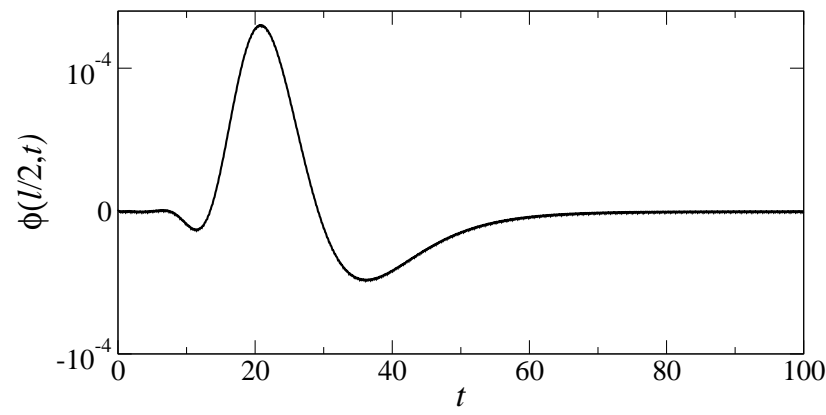

FIG. 2. The magnitude of the disturbance in the middle of a large domain, $l=25$, under LQR control. Exponential asymptotic decay is preceded by a transient, amplifying the initial disturbance (white noise with standard deviation $\sigma=10^{-10}$ ) by six orders of magnitude.

$\lambda_{2}^{\prime}=\cdots=\lambda_{s}^{\prime}=\Lambda$, where $\Lambda$ is some negative number, the product in the numerator of (8) reduces to $\left(\lambda_{m}-\right.$ $\Lambda)^{s}$. Expressing $s$ through $l$ we see that the coefficients $K_{m}$ grow exponentially fast with $l$. The leading order behavior for large $l$ is given by

$$
K_{m} \sim \exp \left[\frac{l}{\pi}\left\{2+\log \left(\lambda_{m}-\Lambda\right)+O\left(\frac{m^{2}}{l^{2}}\right)\right\}\right] .
$$

This exponential growth is clearly seen in Fig. 11. Both here and throughout the paper we use and compare two different control methods. In linear-quadratic (LQR) control [7] the system (4) is truncated to 64 Fourier modes and a set of $K_{m}$ that minimizes a quadratic form in $\Phi$ is sought numerically. In pole placement (PP) the feedback gain is calculated directly from (8), where we change all unstable eigenvalues to $\Lambda=-0.5$ and leave the stable ones unchanged. (The choice of $\Lambda$ is somewhat arbitrary and is chosen to roughly correspond to the average of the first $s$ eigenvalues produced by LQR). Both control laws show the same scaling of $K$ with $l$.

The exponential growth of the control signal suggests transient behavior, a sign of nonnormality. Indeed, a small initial disturbance inside the domain will create a large control perturbation at the right boundary, $x=l$. If the feedback is designed properly, this perturbation will eventually (after propagating through the system) cancel the initial disturbance, thereby making the system asymptotically stable. However, asymptotic decay will be preceded by a transient whose magnitude grows with the feedback gain $K$. Numerical simulation of the linearized GLE does indeed show a large transient (see Fig. 2).

The scaling of both the transient amplification and the control signal can also be understood qualitatively. Since the propagation of perturbations is diffusive, it will take a time $\tau$ roughly proportional to the size of the system, $l$, for the control signal to travel from the right boundary to the left one, suppressing the disturbances inside the domain. Since the system is locally unstable, a disturbance near the left boundary will grow uncontrolled 
during this time interval. The exponential growth of the disturbance will result in its amplification by a factor $\exp \left(\lambda_{1} \tau\right) \sim \exp (\beta l)$. To suppress the amplified disturbance, we need to apply a control perturbation at least as large as the disturbance itself, which requires exponential (with $l$ ) growth in the feedback gain.

Next, let us consider how nonnormality arises in our system. The evolution matrix for the controlled dynamics can be written in the form $M=A+B K^{\dagger}$, where $A$ is the diagonal (and hence normal) matrix that describes the dynamics of the unperturbed system, $A_{m m}=\lambda_{m}$, and $B$ and $K$ are vectors with elements $(-1)^{m+1}$ and $K_{m}$, respectively. Clearly $B K^{\dagger}$ is not a normal matrix, so the sum $A+B K^{\dagger}$ is not normal either. In fact, when all unstable eigenvalues are chosen equal, the matrix $M$ is not even diagonalizable. In that case, one can convert $M$ into the Jordan normal form

$$
J(M)=S^{-1} M S=\left(\begin{array}{cc}
J_{1} & 0 \\
0 & J_{2}
\end{array}\right)
$$

with $J_{1}$ being an $s \times s$ Jordan block with eigenvalue $\Lambda$, $J_{2}$ a diagonal matrix with $\lambda_{s+1}, \lambda_{s+2}, \cdots$ on the diagonal, and $S$ the respective coordinate transformation. This means that the eigenvectors of $M$ corresponding to the Jordan block $J_{1}$ coincide, $e_{1}=e_{2}=\cdots=e_{s}$. The solution of the linear system (何) in this case is constructed using the generalized eigenvectors $e_{p}^{\prime}$, such that $M e_{p}^{\prime}=$ $\Lambda e_{p}^{\prime}+e_{p-1}^{\prime}$ for $p=2, \cdots, s$ and $e_{1}^{\prime}=e_{1}$. Specifically,

$$
\begin{aligned}
\Phi(t) & =\sum_{p=1}^{s}\left[\sum_{m=0}^{s-p} c_{p+m} \frac{t^{m}}{m !}\right] \exp (\Lambda t) e_{p}^{\prime} \\
& +\sum_{p=s+1}^{\infty} c_{p} \exp \left(\lambda_{p} t\right) e_{p},
\end{aligned}
$$

where $c_{1}, c_{2}, \cdots$ are integration constants which have to be chosen to satisfy the initial condition. The result for the individual Fourier coefficients can be written more conveniently using the elements of the transformation matrix $S$ :

$$
\begin{aligned}
\Phi_{n}(t) & =\sum_{p=1}^{s} S_{n, p}\left[\sum_{m=0}^{s-p} c_{p+m} \frac{t^{m}}{m !}\right] \exp (\Lambda t) \\
& +\sum_{p=s+1}^{\infty} S_{n, p} c_{p} \exp \left(\lambda_{p} t\right) .
\end{aligned}
$$

By looking at (11) or 12) one can clearly see that the solution $\phi(x, t)$ grows as a polynomial of order $s-1$ before exponential decay at a rate $\Lambda$ finally takes over.

In the above analysis we required the first $s$ eigenvalues to be equal. What happens in the typical case when these eigenvalues are close, but not equal? First of all, as (8) shows, the coefficients $K_{m}$ of the feedback gain will still grow exponentially with the size of the system. The matrix $M$ will remain strongly nonnormal,

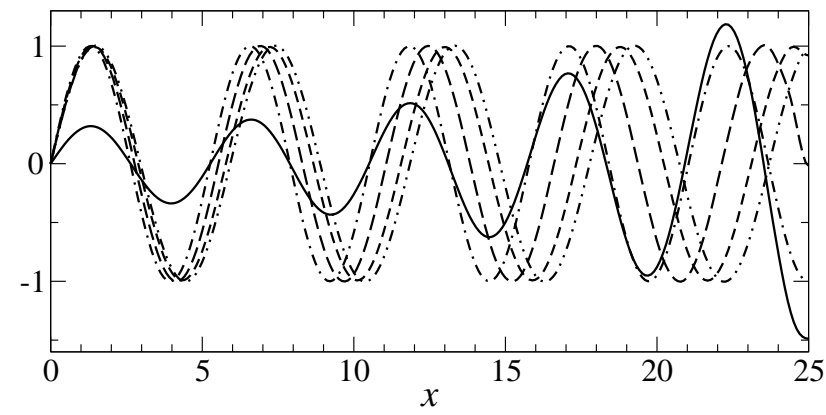

FIG. 3. First five eigenfunctions of the controlled system for $l=25$. The evolution matrix $M$ was calculated using LQR and has all eigenvalues distinct.

but will become diagonalizable. Therefore, all eigenvectors of $M$ will be distinct, but the first $s$ will be closely aligned. This can be argued in the following way. Since $K_{m}$ quickly grows with increasing $\left|\lambda_{m}^{\prime}\right|$, while the size of the largest possible perturbation is usually severely restricted by practical limitations as well as nonlinearities, the new eigenvalues have to be chosen in a small strip of negative values, say $(-\epsilon, 0)$. Therefore, the increase in the system size will force progressively more eigenvalues to lie in this strip, making the difference between successive eigenvalues shrink at least as fast as $\epsilon / l$. As we have seen previously, setting $s$ eigenvalues equal produces an $s \times s$ Jordan block, which causes $s$ eigenvectors to merge. Since $s$ is arbitrary, such merging will occur for any number of eigenvectors corresponding to identical eigenvalues. As a result, pairs of successive eigenvalues will continuously approach each other, aligning the respective eigenvectors. (The continuity can be checked by a straightforward application of perturbation theory.) Fig. 3 shows that already for $l=25$ the first five eigenfunctions are very closely aligned: all have a nearly sinusoidal shape with either 8 or 9 nodes, i.e., almost coincide with the first stable (unperturbed) eigenfunction $f_{9}(x)=\sin (8.5 \pi x / l)$ which has 8 nodes.

Finally, it is useful to derive the quantitative result for transient amplification,

$$
\gamma \equiv \max _{t, \Phi(0)} \frac{\|\Phi(t)\|_{2}}{\|\Phi(0)\|_{2}}=\max _{t}\|\exp (M t)\|_{2},
$$

because this is the ultimate measure that determines when modal analysis and linear control break down. Let us again assume that all unstable eigenvalues are made equal and analyze the structure of the solution (12) more carefully. The transient occurs because each of the terms $\left(t^{m} / m !\right) \exp (\Lambda t)$ first grows as $t^{m}$ and then decays as $\exp (\Lambda t)$, reaching the maximal value at $t_{m}=-m / \Lambda$. This maximal value is given by

$$
\frac{\left(t_{m}\right)^{m}}{m !} \exp \left(\Lambda t_{m}\right) \sim \frac{1}{\sqrt{2 \pi m}|\Lambda|^{m}}
$$

so for small $|\Lambda|$ the term with $m=s-1$ dominates (12). 
A good approximation for the transient amplification $\gamma$ can, therefore, be obtained by picking the initial state $\Phi(0)$ that corresponds to setting $c_{m}=\delta_{m, s}$ and calculating the maximum of the ratio $\|\Phi(t)\|_{2} /\|\Phi(0)\|_{2}$ :

$$
\gamma \sim \sqrt{\frac{\sum_{n=1}^{s} S_{n, 1}^{2}}{\sum_{n=1}^{s} S_{n, s}^{2}}} \max _{t} \frac{t^{s-1} e^{\Lambda t}}{(s-1) !},
$$

where we only keep terms with $p=1$. It should be noted that the chosen initial state corresponds to a "near optimal" disturbance, rather than the "optimal" disturbance producing the largest transient amplification 任.

If the matrix $\mathrm{S}$ is normalized such that $S_{1, s}=1$, it can be shown that, for arbitrary $n, S_{n, s}=\delta_{n, 1}$ and

$$
S_{n, 1}=(-1)^{s+n} \frac{\left(\lambda_{1}-\Lambda\right)^{s}}{\lambda_{n}-\Lambda} \frac{\prod_{k=2}^{s}\left(\lambda_{k}-\Lambda\right)}{\prod_{k=2}^{s}\left(\lambda_{1}-\lambda_{k}\right)} .
$$

Since all $S_{n, 1}$ scale in the same way, (15) gives

$$
\gamma \sim \frac{\left|S_{1,1}\right|}{|\Lambda|^{s-1}}
$$

In order to perform the calculation of the leading order behavior of $S_{1,1}$ we assume that $\Lambda=1-[(p-1 / 2) \pi / l]^{2}$, where $p$ is some integer, in which case (16) gives

$$
\left|S_{1,1}\right|=\frac{\left(\lambda_{1}-\Lambda\right)^{s-1}(p+s-1) !}{(p-s-1) ! p(p-1) s !(s-1) !} .
$$

We can now re-express $p$ in terms of $\Lambda$ thus extrapolating between the known expressions for $\Lambda$ 's corresponding to integer $p$ 's. At the leading order we again obtain exponential growth with the system size:

$$
\gamma \sim \exp \left[\frac{l}{\pi}\left\{\log \frac{\Lambda-1}{\Lambda}+\log \frac{(\alpha+1)^{\alpha+1}}{(\alpha-1)^{\alpha-1}}\right\}\right],
$$

where $\alpha \equiv \sqrt{1-\Lambda}$.

As Fig. 4 shows, the numerically calculated transient amplification factor does indeed grow exponentially fast and is rather insensitive to the way the feedback gain is calculated. The slope is seen to be slightly different from the one predicted by (19). This is to be expected. First, we only consider the terms in (12) for which $m=s-p$. While that gives a correct leading order result for small $|\Lambda|$, for $\Lambda=-0.5$ the contribution from $m=s-p-1$ is about half that from $m=s-p$ and more terms might need to be considered. Second, (19) is the asymptotic result for (15) and is valid only in the limit of large $l$ (here for $l \gtrsim 20$ ). On the other hand, the numerical accuracy in calculating the matrix norm decreases rapidly with $l$. This is in fact a numerical fingerprint of nonnormality. The results found using standard numerical routines are getting rather inaccurate for strongly nonnormal matrices, e.g., for large $l$ (in our case also for $l \gtrsim 20$ ).

The large transient amplification makes the system extremely susceptible to noise, as noise is amplified by feedback before being suppressed. In order for linear control

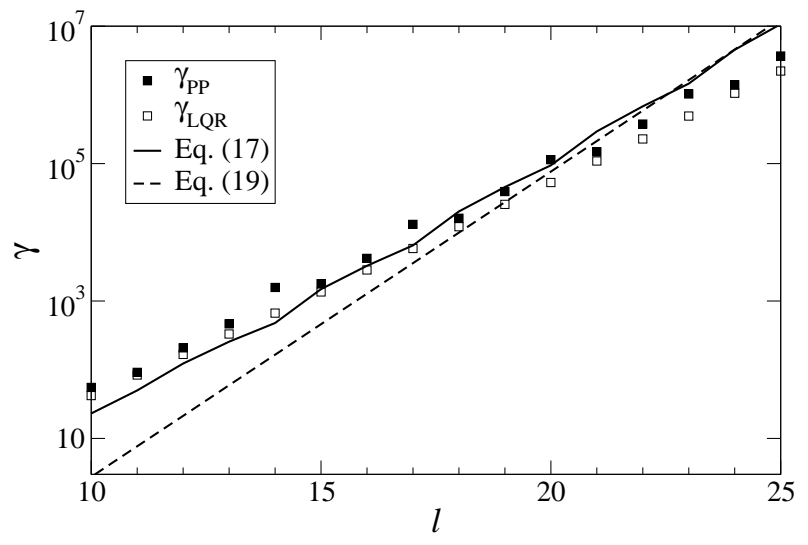

FIG. 4. Transient amplification $\gamma$ as a function of $l$. The squares show the values calculated numerically using (13).

to work, the magnitude of the nonlinear terms has to be smaller than the magnitude of the linear terms. Comparison of their relative magnitude can be used to estimate when noise will start to interfere with control. For instance, for cubic nonlinearity in the GLE, using the argument of Egolf and Socolar [3] we obtain that the largest magnitude of noise, $\sigma$, tolerated by linear control should scale like $\gamma^{-3 / 2}$. However, our numerical calculations produce different scaling. This disagreement is currently under investigation.

To conclude, we have shown that the application of spatially localized control to spatially extended systems renders the system nonnormal. The degree of nonnormality quickly increases with the size of the system due to the close alignment of a progressively larger number of originally unstable eigenfunctions. Increasing nonnormality leads to transient amplification which quickly grows with the size of the system, thus imposing strict limitations on the density of controllers required to control a system of a given size in the presence of noise or truncation errors.

[1] R. O. Grigoriev, Physica D 140, 171 (2000).

[2] R. O. Grigoriev, M. C. Cross, and H. G. Schuster, Phys. Rev. Lett. 79, 2795 (1997)

[3] D. A. Egolf and J. E. S. Socolar, Phys. Rev. E 57, 5271 (1998).

[4] B. F. Farrell and P. J. Ioannou, J. Atmospheric Sci. 53, 2025 (1996).

[5] L. N. Trefethen, A. E. Trefethen, S. C. Reddy, and T. A. Driscoll, Science 261, 578 (1993).

[6] J, S, Baggett, T. A. Driscoll, L. N. Trefethen, Phys. Fluids 7, 833 (1995).

[7] P. Dorato, C. Abdallah and V. Cerrone, Linear-Quadratic Control (Prentice Hall, New Jersey, 1995) 